\title{
Training surgeon status is not associated with an increased risk of early or late mortality after isolated aortic valve replacement surgery
}

\author{
Akshat Saxena ${ }^{1}$, Diem Dinh ${ }^{2}$, Julian A. Smith ${ }^{3,4}$, Christopher M. Reid ${ }^{2}$, \\ Gilbert Shardey ${ }^{5}$, Andrew E. Newcomb ${ }^{1,6}$
}

${ }^{1}$ Department of Cardiothoracic Surgery, St Vincent's Hospital Melbourne, Fitzroy, Victoria, Australia ${ }^{2}$ Department of Epidemiology and Preventative Medicine, Monash University, Prahran, Victoria, Australia ${ }^{3}$ Department of Surgery, Monash University, Monash Medical Centre, Clayton, Victoria, Australia ${ }^{4}$ Department of Cardiothoracic Surgery, Monash Medical Centre, Clayton, Victoria, Australia

${ }^{5}$ Cabrini Medical Centre, Malvern, Victoria, Australia

${ }^{6}$ Department of Surgery, University of Melbourne,

St Vincent's Hospital Melbourne, Fitzroy, Victoria, Australia

\begin{abstract}
Background: Few studies have addressed the effect of "trainee surgeon" status on outcomes after isolated aortic valve replacement (AVR).

Methods and Results: A retrospective analysis of data, collected by the Australasian Society of Cardiac and Thoracic Surgeons Cardiac Surgery Database Program between June 2001 and December 2009 was performed. Patient demographics, intra-operative characteristics and early morbidity were compared between trainee and staff cases. Multivariate analyses were used to determine the independent association of training status with 30-day and late mortality. Isolated AVR was performed in 2747 patients; of these, 369 (13.4\%) were by trainees. Compared to staff cases, trainee cases were less likely to present with renal failure $(1.1 \%$ vs. $3.7 \%, p=0.010)$ or in a critical preoperative state $(1.4 \%$ vs. $3.7 \%, p=0.020)$. The mean EuroSCORE was lower in trainee patients, compared to staff patients (8.11 $\pm 2.80 \mathrm{vs.} 8.81 \pm 3.09, p<0.001)$. Trainee cases had longer mean perfusion (117.9 min vs. $98.9 \mathrm{~min}, p<0.001)$ and cross-clamp (88.8 min vs. $73.2 \mathrm{~min}, p<0.001)$ times. The incidence of early complications was similar between the two groups, except for post-operative myocardial infarction $(1.1 \%$ vs. $0.3 \%, p=0.008)$ and red blood cell transfusion (43.9 vs. $40.0 \%, p=0.006)$. On multivariate analysis, trainee status was not associated with an increased risk of 30 -day mortality (2.2\% vs. $2.4 \%, p=0.823)$. Moreover, there was no significant difference in long-term outcomes and 5-year survival was comparable in both groups (89.9\% vs. $84.8 \%, p=0.274$ ).

Conclusions: Isolated AVR can be safely and effectively performed by trainee surgeons who are strictly supervised in the operating theatre especially during the technically complex parts of the procedure. (Cardiol J 2014; 21, 2: 183-190)
\end{abstract}

Key words: cardiac surgery, aortic valve replacement, trainee, resident, mortality, morbidity, survival

Address for correspondence: Dr Andrew E. Newcomb, Department of Cardiothoracic Surgery, St Vincent's Hospital Melbourne, 41 Victoria Parade, Fitzroy, 3065, Victoria, Australia, tel: +61 39288 2151, fax: +61 39288 4616, e-mail: andrew.newcomb@svhm.org.au

Received: 14.04.2013 Accepted: 09.06.2013 


\section{Introduction}

The outcomes following cardiac operations performed by surgeons-in-training have come under increased scrutiny in recent years. Particularly in the last 2 decades, concerns have been raised that the exposure of trainees to operations has steadily declined in both quality and quantity [1]. A number of factors have been implicated. Firstly, with the rapid ageing population of Western countries, cardiac operations are becoming increasingly complex leading to reluctance amongst surgeons to offer operative opportunities to trainees. Secondly, many training programs around the world have restricted working hours through legislation $[2,3]$. Thirdly, it can be argued that an increase in privately funded cardiac operations, even in countries with a robust public health care system such as Australia, has reduced training opportunities for trainees given the expectation that only trained surgeons perform procedures on privately insured patients. Finally, an increased level of scrutiny on the performance of individual cardiac surgeons secondary to the rise in database participation and public reporting of surgical outcomes may well have translated into decreased opportunities for trainee surgeons $[4,5]$.

Previous studies have demonstrated promising early outcomes after cardiac surgery performed by surgeons-in-training [5-16]. The majority of studies have shown that provided careful supervision, training status does not affect 30-day mortality. There is significantly less data on long-term outcomes, although some studies have shown that excellent long-term outcomes can be achieved by trainees [5, 15-17]. For aortic valve replacement (AVR) providing adequate training opportunities is critical given that this procedure is generally more technically challenging than isolated coronary artery bypass graft (CABG) and is performed less often. Unfortunately, the majority of published series have collectively analyzed different types of cardiac surgery together or addressed outcomes following CABG. As such, there is a paucity of data on the impact of training on outcomes after AVR. Moreover, many studies which have evaluated the impact of AVR have often not reported long-term outcomes. The purpose of this study, therefore, is to evaluate the short and long term outcomes of isolated aortic valve replacement procedures performed by trainee surgeons using the contemporary, multi-institutional Australasian Society of Cardiac and Thoracic Surgeons (ASCTS) National Cardiac Surgery Database.

\section{Methods}

All patients undergoing isolated AVR between 1 June 2001 and 31 December 2009 at hospitals in Australia participating in the ASCTS Cardiac Surgery Database were included in this study. Patients having concomitant valve surgery or other concurrent cardiac surgical procedures were excluded from this study. All 6 Victorian public hospitals which perform adult cardiac surgery - The Royal Melbourne Hospital, The Alfred Hospital, Monash Medical Centre, The Geelong Hospital, Austin Hospital, and St Vincent's Hospital Melbourne - were involved in the prospective data collection during the entire period. Additionally, 14 cardiac surgical units from South Australia, New South Wales, and Queensland have entered the database project in the last 30 months of the study period and contributed $42.4 \%$ of the total patient numbers.

The ASCTS database contained detailed information on patient demographics, pre-operative risk factors, operative details, post-operative hospital course, and morbidity and mortality outcomes. These data were collected prospectively using a standardized dataset and definitions. Data collection and audit methods have been previously described [18]. In the State of Victoria, the collection and reporting of public hospital cardiac surgery data is compulsory and mandated by the State Government; hence it is all-inclusive. Data validation has been a major focus since the establishment of the ASCTS database. The data are subjected to both local validation and an external data quality audit program, which is performed on site to evaluate the completeness (defined as $<1 \%$ missing data for any variable) and accuracy (97.4\%) of the data held in the combined database. Completeness of the database was $96 \%$. Audit outcomes are used to assist in further development of appropriate standards. The Ethics Committee of each participating hospital had previously approved the use of de-identified patient data contained within the database for research and waived the need for individual patient consent.

In the current study, the preoperative characteristics, early outcomes, and long-term survival of patients operated on by trainee surgeons were compared to those operated on by staff (consultant) surgeons. Trainees consisted of junior surgeons who did not have admitting rights at their hospital and were not ultimately responsible for the operation. Trainee surgeries were those in which the trainee performed the entire operation as the primary surgeon or the majority of the critical parts of the operations, in par- 
ticular, the valve replacement. In the former situation, staff surgeon supervision varied depending on the level of training of the trainee surgeon, ranging from the staff surgeon assisting the trainee throughout the surgeries to the staff surgeon being available, but not directly present in the operating room.

Per our database, trainee surgeons were subdivided into 3 groups. First, senior registrars were trainees who were accepted into the Royal Australasian College of Surgeons training program and who had passed the mandatory board examinations but were still completing formal training time. Second, accredited registrars were trainees who were formally accepted into the Royal Australasian College of Surgeons training program but had not completed the mandatory board examinations. These trainees were in postgraduate years 3 to 8 . Overseas fellows, who constitute group 3 , had undergone cardiothoracic surgery training in a country other than Australia and were completing their training in Australia. The Royal Australasian College of Surgeons has set the criteria for minimum operative experience in cardiothoracic surgery. They require trainees to assist with 400 CABGs and perform 70 , assist with 80 AVRs and perform 10 , assist with 30 mitral valve procedures and perform 5 . The total operative experience must be recorded in the trainee logbook, which is assessed every 6 months by the Board of Cardiothoracic Surgery. A more thorough description of minimum operative experience has been provided.

Fourteen early post-operative outcomes were analyzed. These were: a) 30-day mortality, defined as death within 30 days of operation; b) In-hospital mortality, defined as death during hospital stay; c) Permanent stroke, defined as a new central neurologic deficit persisting for $>72 \mathrm{~h}$; d) Transient stroke, defined as a new transient neurologic deficit that resolves completely within $72 \mathrm{~h}$ (transient ischemic attack or reversible ischemic neurologic deficit); e) Postoperative acute myocardial infarction, defined as at least 2 of the following: enzyme level elevation, new cardiac wall motion abnormalities, or new $\mathrm{Q}$ waves on serial electrocardiograms; f) New renal failure, defined as at least 2 of the following: serum creatinine increased to more than $200 \mu \mathrm{mol} / \mathrm{L}$, doubling or greater increase in creatinine when compared with the preoperative value, or new requirement for dialysis or hemofiltration; g) Prolonged ventilation ( $>24 \mathrm{~h}$ ); h) Multi-system failure; defined as concurrent failure of 2 or more of the cardiac, respiratory or renal systems for at least 48 h; i) Red blood cell transfusion; j) Gastrointestinal (GI) complications, defined as post-operative occurrence of any GI complication; k) Deep sternal infection involving muscle and bone as demonstrated by surgical exploration and one of the following: positive cultures or treatment with antibiotics; 1) Return to the operating theatre for any cause; and $\mathrm{m})$ Return to the operating theatre for bleeding.

To assess the impact of trainee status on each early outcome, logistic regression analysis was used to adjust for 18 preoperative patient variables, with the outcome as the dependent variable (variables in Table 4). Long-term survival status was obtained from the Australian National Death Index. The closing date was March 18, 2010. A Kaplan-Meier estimate of survival was obtained. Differences in long-term survival were assessed by the log-rank test. The role of sex in long-term survival was assessed by constructing a Cox proportional hazards model using sex and other preoperative patient characteristics as variables. Continuous variables are presented as mean \pm one standard deviation. The Mann-Whitney U test was used to compare two groups of continuous variables. The $\chi^{2}$ test was used to compare groups of categorical variables. All calculated values of $\mathrm{p}$ were 2 -sided, and $\mathrm{p}<0.05$ was considered significant. Statistical analysis was performed using SPSS ${ }^{\oplus}$ for Windows version 17.0 (SPSS Inc., Chicago, Illinois).

\section{Results}

\section{Patient demographics and pre-operative variables}

Isolated aortic valve replacement was undertaken in 2747 patients; of these, trainees performed $369(13.4 \%)$ surgeries. Of these, 118 were performed by a senior registrar, 117 by an accredited registrar and 134 by an overseas fellow. Pre-operative and demographic characteristics of trainee and staff patients are provided in Table 1 . The mean age of staff patients was $68.2 \pm 11.4$ years compared to $68.5 \pm 13.3$ years for trainee patients $(\mathrm{p}=0.693)$. This was not statistically significant. Trainee patients were less likely to present with renal failure $(1.1 \%$ vs. $3.7 \%, \mathrm{p}=0.010)$ or a critical pre-operative state $(1.4 \%$ vs. $3.7 \%, \mathrm{p}=0.020)$. The mean EuroSCORE was lower in trainee patients, compared to staff patients $(8.81 \pm 3.09$ vs. $8.11 \pm$ $\pm 2.80, \mathrm{p}<0.001)$. Other characteristics were similar between both groups.

\section{Intra-operative data}

Intra-operative data are summarized in Table 2. There were some significant differences in intra-operative variables between the two groups. Trainees had longer mean cardiopulmonary bypass 
Table 1. Pre-operative characteristics and patient demographics, stratified by proceduralist.

\begin{tabular}{lccc}
\hline Preoperative variables & Staff & Trainee & P \\
\hline Total number of patients & 2378 & 369 & - \\
Age [years] & $68.2(11.4 \%)$ & $68.5(13.3 \%)$ & 0.693 \\
Female & $995(41.8 \%)$ & $155(42.0 \%)$ & 0.953 \\
Chronic obstructive pulmonary disease & $344(14.6 \%)$ & $57(15.4 \%)$ & 0.619 \\
Diabetes mellitus & $528(22.2 \%)$ & $73(19.8 \%)$ & 0.311 \\
Hypercholesterolemia & $1175(49.4 \%)$ & $186(50.4 \%)$ & 0.780 \\
Hypertension & $1561(65.6 \%)$ & $249(67.5 \%)$ & 0.522 \\
Cerebrovascular disease & $243(10.2 \%)$ & $35(9.5 \%)$ & 0.657 \\
Peripheral vascular disease & $154(6.5 \%)$ & $18(4.9 \%)$ & 0.236 \\
Renal failure & $87(3.7 \%)$ & $4(1.1 \%)$ & 0.010 \\
Recent myocardial infarction & $29(1.2 \%)$ & $6(1.6 \%)$ & 0.517 \\
History of congestive heart failure & $901(37.9 \%)$ & $131(35.5 \%)$ & 0.366 \\
Coronary artery disease & $221(9.3 \%)$ & $24(6.5 \%)$ & 0.072 \\
Left ventricular ejection fraction: & - & - & 0.054 \\
$\quad$ Normal (> 0.60) [\%] & $1438(60.5 \%)$ & $253(68.6 \%)$ & - \\
Mild (0.45-0.60) [\%] & $530(22.3 \%)$ & $74(20.1 \%)$ & - \\
Moderate (0.30-0.45) [\%] & $221(9.3 \%)$ & $30(8.1 \%)$ & - \\
Severe (<0.30) [\%] & $109(4.6 \%)$ & $9(2.4 \%)$ & - \\
Obesity & $834(35.1 \%)$ & $137(37.1 \%)$ & 0.442 \\
New York Heart Association classification & - & - & 0.218 \\
Class I & $419(17.6 \%)$ & $49(13.3 \%)$ & - \\
Class II & $868(36.5 \%)$ & $146(40.0 \%)$ & - \\
Class III & $866(36.4 \%)$ & $138(37.4 \%)$ & - \\
Class IV & $176(7.4 \%)$ & $27(7.3 \%)$ & - \\
Status & - & - & - \\
Elective & $1964(82.6 \%)$ & $318(86.2 \%)$ & - \\
Urgent/Emergency/Salvage & $414(17.4 \%)$ & $51(13.8 \%)$ & 0.020 \\
Critical preoperative state & $88(3.7 \%)$ & $5(1.4 \%)$ & - \\
EuroSCORE (additive) & $8.81(3.09 \%)$ & $8.11(2.80 \%)$ & \\
\hline
\end{tabular}

Table 2. Intra-operative characteristics, stratified by proceduralist.

\begin{tabular}{lccc}
\hline Preoperative variables & Staff & Trainee & P \\
\hline Total number of patients & 2378 & 369 & - \\
Cardiopulmonary bypass time $[\mathrm{min}]$ & $98.88(40.51 \%)$ & $117.93(35.02 \%)$ & $<0.001$ \\
Aortic cross-clamp time $[\mathrm{min}]$ & $73.24(26.11 \%)$ & $88.75(22.36 \%)$ & $<0.001$ \\
Type of prosthesis: & - & - & 0.543 \\
$\quad$ Bioprosthesis & $1578(66.4 \%)$ & $247(66.9 \%)$ & - \\
Mechanical valve & $724(30.4 \%)$ & $110(29.8 \%)$ & - \\
Other & $21(0.9 \%)$ & $1(0.3 \%)$ & - \\
Valve size & $23.00(2.16 \%)$ & $22.86(2.24 \%)$ & 0.334 \\
\hline
\end{tabular}

$(117.93 \pm 35.02 \min$ vs. $98.88 \pm 40.51 \mathrm{~min}, \mathrm{p}<$ $<0.001)$ and aortic cross-clamp times $(88.75 \pm$ \pm 22.36 min vs. $73.24 \pm 26.11 \mathrm{~min}, \mathrm{p}<0.001)$. There was no difference in the type or size of implanted valve between the two groups.

\section{Early outcomes}

Overall 30-day mortality and in-hospital mortality were $2.3 \%$ and $2.7 \%$, respectively. The unadjusted 30 -day mortality rate was $2.2 \%$ in trainee patients and $2.4 \%$ in staff patients. This difference was 
Table 3. Predictors for 30 -day and late mortality in entire cohort $(n=2747)$.

\begin{tabular}{lcccc}
\hline Preoperative variables & \multicolumn{2}{c}{30 -day mortality } & & \multicolumn{2}{c}{ Late mortality } \\
\cline { 2 - 3 } & $\begin{array}{c}\text { Odds ratio (95\% } \\
\text { confidence interval) }\end{array}$ & & $\begin{array}{c}\text { Odds ratio (95\% } \\
\text { confidence interval) }\end{array}$ \\
\hline Age [years] & $1.07(1.04-1.11)$ & $<0.001$ & $1.07(1.05-1.08)$ & $\mathbf{P}$ \\
Trainee surgeon status & $1.10(0.48-1.70)$ & 0.823 & $0.82(0.57-1.17)$ & 0.274 \\
Female & $0.95(0.53-1.70)$ & 0.851 & $0.94(0.74-1.21)$ & 0.637 \\
Chronic obstructive pulmonary disease & $1.88(1.03-3.44)$ & 0.040 & $1.34(1.01-1.76)$ & 0.041 \\
Diabetes mellitus & $1.99(1.10-3.60)$ & 0.023 & $1.66(1.27-2.18)$ & $<0.001$ \\
Hypercholesterolemia & $1.26(0.69-2.30)$ & 0.445 & $1.02(0.79-1.30)$ & 0.909 \\
Hypertension & $0.67(0.36-1.26)$ & 0.214 & $0.86(0.66-1.12)$ & 0.266 \\
Cerebrovascular disease & $1.90(0.96-3.76)$ & 0.067 & $1.61(1.17-2.23)$ & 0.004 \\
Peripheral vascular disease & $1.35(0.58-3.15)$ & 0.483 & $1.07(0.70-1.63)$ & 0.770 \\
Renal failure & $5.09(1.67-15.5)$ & 0.004 & $3.15(1.85-5.34)$ & $<0.001$ \\
Critical preoperative state & $0.59(0.15-2.32)$ & 0.452 & $1.32(0.75-2.31)$ & 0.340 \\
Coronary artery disease & $0.60(0.24-1.55)$ & 0.293 & $1.03(0.70-1.53)$ & 0.883 \\
Obesity & $0.91(0.48-1.72)$ & 0.780 & $0.96(0.74-1.26)$ & 0.774 \\
Recent myocardial infarction & $1.49(0.38-5.84)$ & 0.567 & $0.74(0.31-1.73)$ & 0.480 \\
History of congestive heart failure [\%] & $2.03(1.10-3.74)$ & 0.023 & $1.52(1.19-1.96)$ & 0.001 \\
Left ventricular ejection fraction $<0.45$ & $1.85(0.99-3.47)$ & 0.054 & $1.49(1.11-1.99)$ & 0.008 \\
New York Heart Association classification III or IV & $1.34(0.73-2.46)$ & 0.352 & $1.29(1.00-1.65)$ & 0.046 \\
Non-elective procedure & $1.95(1.05-3.62)$ & 0.034 & $1.14(0.85-1.53)$ & 0.396 \\
\hline
\end{tabular}

Table 4. Early outcomes, stratified by proceduralist.

\begin{tabular}{|c|c|c|c|c|}
\hline Outcome & Staff & Trainee & $\mathbf{P}$ & $\begin{array}{c}\text { Trainee } \\
\text { Adjusted odds ratio } \\
\text { (95\% confidence } \\
\text { interval) }\end{array}$ \\
\hline Permanent stroke & $32(1.3 \%)$ & $2(0.5 \%)$ & 0.383 & $0.52(0.12-2.26)$ \\
\hline Transient stroke & $10(0.4 \%)$ & $2(0.5 \%)$ & 0.697 & $1.36(0.29-6.37)$ \\
\hline Post-operative myocardial infarction & $8(0.3 \%)$ & $4(1.1 \%)$ & 0.008 & $6.66(1.64-27.08)$ \\
\hline New renal failure & $143(6.0 \%)$ & $13(3.5 \%)$ & 0.138 & $0.64(0.35-1.16)$ \\
\hline Deep sternal wound infection & $6(0.3 \%)$ & $1(0.3 \%)$ & 0.776 & $1.30(0.22-7.86)$ \\
\hline Septicemia & $26(1.1 \%)$ & $7(1.9 \%)$ & 0.036 & $2.63(1.06-6.50)$ \\
\hline Gastrointestinal complications & $38(1.6 \%)$ & $7(1.9 \%)$ & 0.279 & $1.59(0.69-3.70)$ \\
\hline Multi-system failure & $29(1.2 \%)$ & $4(1.1 \%)$ & 0.701 & $1.24(0.42-3.65)$ \\
\hline Red blood cell transfusion & $948(40.0 \%)$ & $162(43.9 \%)$ & 0.006 & $1.40(1.10-1.79)$ \\
\hline Prolonged ventilation & $189(7.9 \%)$ & $32(8.7 \%)$ & 0.256 & $1.27(0.84-1.92)$ \\
\hline Return to theatre & $196(8.2 \%)$ & $29(7.9 \%)$ & 0.698 & $1.09(0.72-1.65)$ \\
\hline Return to theatre for bleeding & $90(3.8 \%)$ & $19(5.1 \%)$ & 0.098 & $1.55(0.92-2.61)$ \\
\hline
\end{tabular}

not significant on univariate analysis $(\mathrm{p}=1.000)$. The logistic regression model predicting 30-day mortality is shown in Table 3. This model has a Hosmer-Lemeshow $\chi^{2}$ statistic of $4.77(\mathrm{p}=0.78)$ and demonstrates that trainee status was not an independent predictor for 30-day mortality $(\mathrm{p}=0.823)$. The independent association of trainee status with other post-operative outcomes is summarized in Table 4. On univariate analysis, trainee patients had a significantly lower mean post-operative length of stay $(9.08 \pm 7.40$ days vs. $10.14 \pm 9.65$ days, $\mathrm{p}=0.043)$ but not intensive care unit stay $(44.58 \pm$ $\pm 71.61 \mathrm{~h}$ vs. $50.58 \pm 86.16 \mathrm{~h}, \mathrm{p}=0.205)$ compared to staff patients.

\section{Late outcomes}

Long-term survival at $1,3,5$, and 7 years post-operatively was higher in trainee patients 


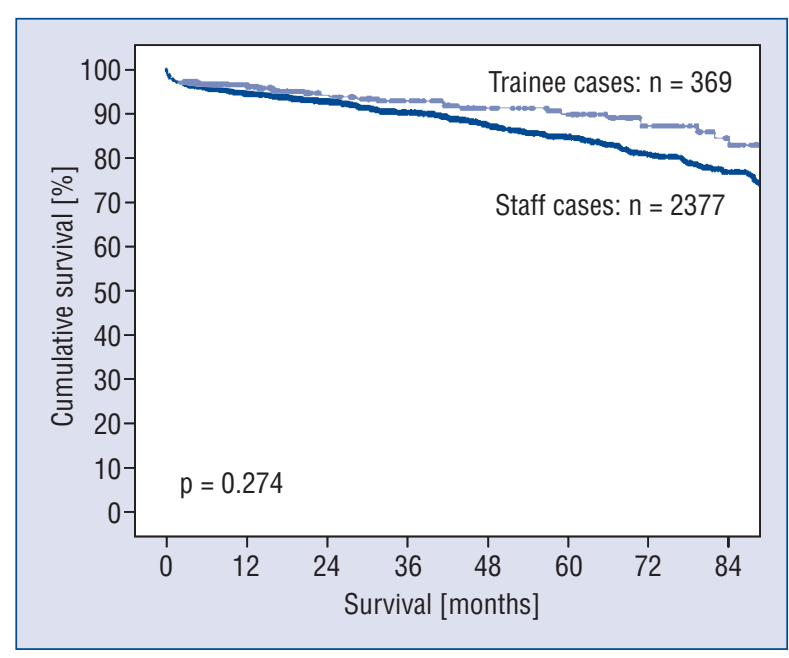

Figure 1. Overall survival of patients after isolated aortic valve replacement, stratified by proceduralist.

compared to staff patients on univariate analysis ( $96.1 \%$ vs. $94.5 \%, 92.9 \%$ vs. $90.3 \%, 89.9 \%$ vs. $84.8 \%$ and $83.0 \%$ vs. $76.9 \%, \mathrm{p}=0.028$ ) (Fig. 1). However, after adjusting for differences in patient variables, there was no difference in survival outcomes between the two groups $(p=0.274)$. A Cox regression model predicting late mortality is summarized in Table 3.

\section{Discussion}

Several previous studies have demonstrated that allowing trainee surgeons to perform cardiac surgery under good supervision is not associated with worse early or late outcomes [5-17]. The majority of these studies have evaluated the outcomes of cardiac surgery in general or isolated CABG procedures. Some studies have evaluated the early outcomes of AVR performed by trainee surgeons but there is a paucity of literature on long-term outcomes [6, 15]. We used the large, multi-institutional ASCTS database to address both early and late outcomes after isolated AVR.

A comparison of pre-operative characteristics between cases performed by residents and staff surgeons demonstrated some differences. Trainees were less likely than staff surgeons to operate on patients with renal failure or in a critical pre-operative state. There was, however, no difference in the incidence of other co-morbidities including peripheral vascular disease, hypertension, cerebrovascular disease, congestive heart failure or diabetes between the two groups. Overall, the mean additive EuroSCORE, which is a surrogate marker for operative risk, was slightly lower in patients for whom the primary surgeon was a trainee (8.11 vs. 8.8, $\mathrm{p}<0.001)$. This indicates that the pre-operative profile of patients who were treated by staff was slightly worse than patients treated by trainees. Some studies have also shown that trainees generally operate on less complex cases $[5,10,16,17]$. Others, however, have shown the converse. Stoica et al. [15] in an analysis of 1054 trainee cases including 118 isolated AVR cases demonstrated that trainee cases were more complex and patients presented with greater co-morbidities. This arose from an expectation at the institution that residents chose cases that were more complex. Despite this there was no increase in hospital mortality in trainee cases compared to staff cases (3.98\% vs. $2.91 \%, \mathrm{p}=0.66)$. The authors argued that exposure to complex cases was beneficial both in terms of residency training and exposure to difficult decision making. Similar findings have been reported elsewhere $[6,19]$. These encouraging findings suggest that case complexity should not preclude trainee surgeons under proper supervision from operating.

Intra-operatively, trainees tended to have longer cardiopulmonary bypass and aortic cross-clamp times. This finding is intuitive and a loss of intraoperative efficiency is an unavoidable part of resident training. This may be of concern, at least theoretically, given the potential risks and deleterious effects associated with perfusion support $[8,20]$, including particulate microembolic [21], platelet and red blood cell effects, increased transfusion requirements [22], coagulation defects, immunosuppression [23], and systemic inflammatory response syndrome [24]. Fortunately, as demonstrated by Haan et al. [8] the increased perfusion and bypass time associated with resident training does not compromise early outcomes.

Our study supports this notion. In fact trainee surgeons achieved excellent early outcomes. Our data showed a 30 -day mortality of $2.2 \%$ and $2.4 \%$ in trainee and staff cases, respectively. This was not statistically significant $(\mathrm{p}=0.274)$. Similar findings have been reported previously, albeit in studies with a significantly small sample size $[6,14,15]$. Baskett et al. [6] evaluated the impact of training status on outcomes in a series of 447 patients who underwent AVR with or without CABG. There was no difference in early mortality between the resident and staff group (3.6\% vs. $2.8 \%$, $\mathrm{p}=0.69)$. Stoica etal. [15] demonstrated no difference in the in-hospital mortality rate between resident and staff surgeons in the entire study population, 
as well as the AVR \pm CABG subgroup. Similar findings have been reported across the spectrum of cardiac surgery procedures, including on-pump CABG $[5,6,8,16]$, off-pump CABG $[10,11]$ and mitral valve surgery $[13,17]$. Our data correlates with these findings and suggests that provided proper supervision, training status does not increase the risk of early mortality after AVR.

A comparison of other early outcomes also demonstrated similar outcomes between the two groups. On univariate analysis, training status was not associated with an increased risk of stroke, new renal failure, multi-system failure, prolonged ventilation or return to theatre. Contrary to the findings of other studies, however, the incidence of post-operative myocardial infarction (PMI) was higher in trainee cases $(1.1 \%$ vs. $0.3 \%, \mathrm{p}=0.008)$ $[6,16]$. This may reflect the longer perfusion and aortic cross-clamp time in trainee cases, which have been previously shown to be risk factors for PMI [25]. Nevertheless, the incidence of PMI in the trainee group is comparable to that reported in many other studies and it may represent a chance finding given that the overall incidence of PMI in the current study was low at only $0.4 \%$ $[6,16,26]$. Our study also showed a significantly higher incidence of red blood cell transfusion in the trainee group $(43.9 \%$ vs. $40.0 \%, \mathrm{p}=0.006)$. The majority of published series, including those which specifically addressed early outcomes, have not analyzed the impact of training status on red blood cell transfusion. This is an important finding given the association of transfusion with poorer early and late outcomes and an increased cost of hospitalization [27, 28]. Septicemia was also significantly more likely to develop in trainee cases (1.9\% vs. $1.1 \%, \mathrm{p}=0.036)$. This finding is worrying given that septicemia portends a poorer outcome. Unfortunately, many published series did not investigate for a possible association between trainee surgeon status and septicemia $[6-9,11]$. Baskett et al. [6] demonstrated a higher incidence of sternal infection but did not specifically evaluate the association between trainee surgeon status and septicemia. This association merits further investigation.

A comparison of long-term outcomes also demonstrated that trainee surgeons achieve equivalent outcomes to staff specialists. Unadjusted 5 -year event-free survival in trainee and staff cases was $55.8 \%$ and $58.6 \%$, respectively. This was not significant on univariate $(\mathrm{p}=0.06)$ or multivariate analysis $(p=0.42)$. In the isolated AVR subgroup, trainee status did not confer an increased risk of late mortality $(\mathrm{p}=0.91)$. Yap et al. [5] demonstrated that trainee status did not compromise long-term survival after isolated CABG surgery. More recently, Shi et al. [17] applied propensity analysis techniques to a group of patients undergoing mitral valve surgery and demonstrated equivalent outcomes between trainees and residents. Our data suggest that this trend also applies to patients undergoing isolated AVR surgery.

Our study has several advantages and disadvantages. The main advantage is that as a contemporary, multi-institutional analysis, our study accurately reflects actual clinical practice. The major disadvantage is that as a retrospective review, there is the potential for selection bias especially because trainee surgeons select or are allocated which cases to perform. Second, we did not treat the allocation of surgeries on an intent-to-treat basis. Consequently, cases in which a staff specialist completed a surgery initiated by the trainee would have almost certainly been regarded as a consultant surgery. Third, although risk adjustment analyses using multivariate analysis were performed, the lack of random treatment allocation means that residual confounding from residual or unrecognized variables cannot be excluded. Fourth, whilst our database allows an analysis of long-term mortality, we are not able to determine the cause of late mortality. Regardless of these limitations, our data strongly implies that training status does not confer a worse early or late outcome in patients undergoing isolated AVR.

\section{Conclusions}

In conclusion, AVR can be safely performed by trainee surgeons under good supervision. Trainee status is not associated with an increased risk of early or late mortality and is associated with a similar incidence of most post-operative complications.

\section{Acknowledgements}

The following investigators, data managers and institutions participated in the ASCTS Database: Alfred Hospital: Pick A, Duncan J; Austin Hospital: Seevanayagam S, Shaw M; Cabrini Health: Shardey G; Geelong Hospital: Morteza M, Bright C; Flinders Medical Centre: Knight J, Baker R, Helm J; Jessie McPherson Private Hospital: Smith J, Baxter H; Hospital: John Hunter Hospital: James A, Scaybrook S; Lake Macquarie Hospital: Dennett B, Jacobi M; Liverpool Hospital: French B, Hewitt N; Mater Health Service Hospital: Diqer AM, Archer J; Monash Medical Centre: Smith J, Baxter H; Prince 
of Wales Hospital: Wolfenden H, Weerasinge D; Royal Melbourne Hospital: Skillington P, Law S; Royal Prince Alfred Hospital: Wilson M, Turner L; St George Hospital: Fermanis G, Redmond C; St Vincent's Hospital, VIC: Yii M, Newcomb A, MackJ, Duve K; St Vincent's Hospital, NSW: Spratt P, Hunter T; The Canberra Hospital: Bissaker P, Butler K; Townsville Hospital: Tam R, Farley A; Westmead Hospital: Costa R, Halaka M.

\section{Funding}

The Australasian Society of Cardiac and Thoracic Surgeons (ASCTS) National Cardiac Surgery Database Program is funded by the Department of Human Services, Victoria, and the Health Administration Corporation (GMCT) and the Clinical Excellence Commission (CEC), NSW.

\section{Conflict of interest: none declared}

\section{References}

1. Wang TT, Ahmed K, Patel VM et al. A competency framework in cardiothoracic surgery for training and revalidation: An international comparison. Eur J Cardiothorac Surg, 2011; 40: 816-825.

2. DeLaRosa J, Thourani VH, Wheatley GH, $3^{\text {rd }}$ et al. Impact of resident duty hour standards on cardiothoracic residents and program directors. Ann Thorac Surg, 2005; 80: 1569-1571.

3. Lim E, Tsui S. Impact of the european working time directive on exposure to operative cardiac surgical training. Eur J Cardiothorac Surg, 2006; 30: 574-577.

4. Keogh B, Spiegelhalter D, Bailey A, Roxburgh J, Magee P, Hilton C. The legacy of bristol: Public disclosure of individual surgeons' results. BMJ, 2004; 329: 450-454.

5. Yap $\mathrm{CH}$, Andrianopoulos N, Dinh TD et al. Short- and midterm outcomes of coronary artery bypass surgery performed by surgeons in training. J Thorac Cardiovasc Surg, 2009; 137: 1088-1092.

6. Baskett RJ, Buth KJ, Legare JF et al. Is it safe to train residents to perform cardiac surgery? Ann Thorac Surg, 2002; 74: 1043 -1048; discussion 1048-1049.

7. Goodwin AT, Birdi I, Ramesh TP et al. Effect of surgical training on outcome and hospital costs in coronary surgery. Heart, 2001; 85: 454-457.

8. Haan CK, Milford-Beland S, O'Brien S et al. Impact of residency status on perfusion times and outcomes for coronary artery bypass graft surgery. Ann Thorac Surg, 2007; 83: 2103-2110.

9. Gulbins H, Pritisanac A, Ennker IC, Ennker J. Safety of a cardiac surgical training program over a twelve-year period. Thorac Cardiovasc Surg, 2007; 55: 494-499.

10. Caputo M, Chamberlain MH, Ozalp F, Underwood MJ, Ciulli F, Angelini GD. Off-pump coronary operations can be safely taught to cardiothoracic trainees. Ann Thorac Surg, 2001; 71: 1215-1219 .

11. Asimakopoulos G, Karagounis AP, Valencia O, Rose D, Niranjan G, Chandrasekaran V. How safe is it to train residents to perform off-pump coronary artery bypass surgery? Ann Thorac Surg, 2006; 81: 568-572.

12. Ascione R, Reeves BC, Pano M, Angelini GD. Trainees operating on high-risk patients without cardiopulmonary bypass: A high-risk strategy? Ann Thorac Surg, 2004; 78: 26-33.

13. Baskett RJ, Kalavrouziotis D, Buth KJ, Hirsch GM, Sullivan JA. Training residents in mitral valve surgery. Ann Thorac Surg, 2004; 78: 1236-1240.

14. Sethi GK, Hammermeister KE, Oprian C, Henderson W. Impact of resident training on postoperative morbidity in patients undergoing single valve replacement. Department of veterans affairs cooperative study on valvular heart disease. J Thorac Cardiovasc Surg, 1991; 101: 1053-1059.

15. Stoica SC, Kalavrouziotis D, Martin BJ et al. Long-term results of heart operations performed by surgeons-in-training. Circulation, 2008; 118: S1-S6.

16. Oo AY, Grayson AD, Rashid A. Effect of training on outcomes following coronary artery bypass graft surgery. Eur J Cardiothorac Surg, 2004; 25: 591-596.

17. Shi WY, Hayward PA, Yap CH et al. Training in mitral valve surgery need not affect early outcomes and midterm survival: A multicentre analysis. Eur J Cardiothorac Surg, 2011; 40: 826-833.

18. Dinh DT, Lee GA, Billah B, Smith JA, Shardey GC, Reid CM. Trends in coronary artery bypass graft surgery in victoria, 2001-2006: Findings from the australasian society of cardiac and thoracic surgeons database project. Med J Aust, 2008; 188: 214-217.

19. Bakaeen FG, Dhaliwal AS, Chu D et al. Does the level of experience of residents affect outcomes of coronary artery bypass surgery? Ann Thorac Surg, 2009; 87: 1127-1133; discussion 1133-1124.

20. Edmunds LH, Jr. Blood-surface interactions during cardiopulmonary bypass. J Card Surg, 1993; 8: 404-410.

21. Taylor KM. Brain damage during cardiopulmonary bypass. Ann Thorac Surg. 1998; 65: S20-S26; discussion S27-S28.

22. Despotis GJ, Filos KS, Zoys TN, Hogue CW, Jr., Spitznagel E, Lappas DG. Factors associated with excessive postoperative blood loss and hemostatic transfusion requirements: A multivariate analysis in cardiac surgical patients. Anesth Analg, 1996; 82: 13-21.

23. Sablotzki A, Welters I, Lehmann N, Menges T, Gorlach G, Dehne M, Hempelmann G. Plasma levels of immunoinhibitory cytokines interleukin-10 and transforming growth factor-beta in patients undergoing coronary artery bypass grafting. Eur J Cardiothorac Surg, 1997; 11: 763-768.

24. Royston D. Systemic inflammatory responses to surgery with cardiopulmonary bypass. Perfusion, 1996; 11: 177-189.

25. Nissinen J, Biancari F, Wistbacka JO et al. Safe time limits of aortic cross-clamping and cardiopulmonary bypass in adult cardiac surgery. Perfusion, 2009; 24: 297-305.

26. Casati V, Guzzon D, Oppizzi M et al.Tranexamic acid compared with high-dose aprotinin in primary elective heart operations: Effects on perioperative bleeding and allogeneic transfusions. J Thorac Cardiovasc Surg, 2000; 120: 520-527.

27. Rawn JD. Blood transfusion in cardiac surgery: A silent epidemic revisited. Circulation, 2007; 116: 2523-2524.

28. Koch CG, Li L, Duncan AI et al. Transfusion in coronary artery bypass grafting is associated with reduced long-term survival. Ann Thorac Surg, 2006; 81: 1650-1657. 